\title{
Energy Efficiency Analysis of the Discrete Time Three-Dimensional Probability CSMA Protocol in WSN
}

\author{
Sheng Jie Zhou ${ }^{1}$, Hong Wei Ding ${ }^{1,2}$, Zhi Jun Yang ${ }^{1}$, Qian Lin Liu ${ }^{1}$ and Meng Yao Wang ${ }^{1}$ \\ ${ }^{1}$ School of Information, Yunnan University, Kunming, Yunnan, China \\ 2E-mail:dhw1964@163.com
}

\begin{abstract}
In the wireless sensor network, energy supply and consumption is the main factors to affect survival time. Introducing the three-dimensional probability CSMA protocol, the paper analyses the system throughput, system delay and energy efficiency with using the average cycle analysis methods. For this protocol, the system time is slotted into a time slot with high channel utilization realized by the choice of three parameters $\mathrm{P} 1, \mathrm{P} 2$ and $\mathrm{P} 3$. The correctness of the theory is verified through the simulation.

Keywords: WSN, three-dimensional probability, CSMA, throughput.
\end{abstract}

\section{Introduction}

Wireless sensor networks (Wireless Sensor Networks, WSN) are deployed in the detection zone through a lot with network nodes which have the wireless communication and computing capabilities. It is able to complete the assigned task of distributed intelligent network system consisting of selforganization of nodes. Between the nodes of a wireless sensor network is a short distance; the general multi-hop wireless communication. The network can be run in a standalone environment. You can also connect to the Internet through a gateway in remote access. Wireless sensor networks originated in military applications, and now, wireless sensor networks are increasingly used in environmental and ecological monitoring, health monitoring, home automation and traffic control, and many other civilian areas ${ }^{[2]}$.

Typically wireless sensor nodes in the network are widely distributed at a larger number, which cannot rely on supplemental batteries as an energy source. In many cases due to constraints, maintenance personnel inaccessible, battery replacement is very difficult or even impossible to replace. And requirements of lifetime for the network are months or even years. So the energy supply has become a major factor affecting network effective survival time and overall network performance. How in the existing energy supply conditions, without affecting system functionality premise, as far as possible to reduce energy consumption, save battery power, maximizing network lifetime wireless sensor networks has become the core issue.

Since each node sends a packet random, in order to reduce collisions, the MAC layer protocol necessary to establish a shared channel access mechanism. Efficient MAC layer protocol is a hot topic for the Ad-hoc networks; the most common MAC layer protocol is carrier sense multiple access (CSMA) and a variety of other mechanisms.

\section{The three-dimensional probability CSMA protocol}

In the proposed protocol, there will be three random events:

a) Event that information packets are sent successfully (U events).

b) Event that information packets collide with each other (the collision appears, $\mathrm{C}$ events).

c) Event that there are no information packets in the channel arrive, the channel is idle (I events) ${ }^{[3]}$.

These three events are forced into: the channel is idle (I events) event, the channel is busy (CU events) and the channel is idle following the CU events (CUI events); the packet is sent successfully or unsuccessfully (combined $\mathrm{C}$ events with $\mathrm{U}$ event, denoted by $\mathrm{CU}$ event); force the $\mathrm{CU}$ events and the CUI events into B events ${ }^{[4]}$.

A cycle period is $T_{n}$. The three events above continuously staggering circulate on the time axis and the cycle is variable ${ }^{T_{n}[5]} . T P$ is the transmission period. Use three-dimensional probability: $P 1, P 2$, $P 3$ to control the period of I events, CU events and CUI events separately. 


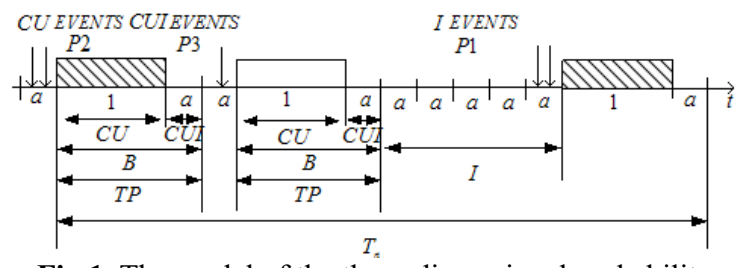

Fig.1. The model of the three-dimensional probability CSMA protocol

According to the protocol, if the channel is idle, then the user decides to send an information packet probability $P 1$; if the channel is busy, the user listens to the channel at probability $P 2$ in the $\mathrm{CU}$ events; in the transportation period, if the channel is the first idle following the $\mathrm{CU}$ events, then the user listens to the channel at probability $P 3{ }^{[6]}$. This control strategy, $P 1, P 2$ and $P 3$ by three-dimensional selection enables the system under different load utilization and throughput is guaranteed.

\section{Analysis of the model}

\subsection{The Analysis of System Throughput}

Before analyze the system performance, first do the following assumptions:

a) The channel is ideal with no noise and interference;

b) The basic unit of the system control clock is $a$, the information packets arrived at time $a$ will transmit at the starting time of the next slot;

c) The channel propagation delay is $a$,the packet length is unit length and is an integral multiple of $a$;

d) The arrival process of channel satisfies the Poisson process whose independent parameter is $G$, each arrival process on the channel is independent of each other;

e) The channel using three-dimensional probability CSMA protocol, the information packets need to be sent at the first slot in the transmission period can always detecting the state of the channel at last moment;

f) During the transmission of information packets, the phenomenon of packet collisions occur inevitably, and continues to be sent after a random time delay, it sends will not produce any adverse effects on the arrival process channel ${ }^{[7]}$.

In a cycle, the average length of time slot that information packet has been successfully sent in a cycle is:

$$
\begin{aligned}
E(U)= & E\left(U_{1}\right)+E\left(U_{2}\right) \\
& =\frac{a p_{1} G e^{-a p_{1} G}}{1-e^{-a p_{1} G}}+\left(a p_{3}+p_{2}\right) G
\end{aligned}
$$

The average length of $B$ event is:

$$
E(B)=E\left(N_{B}\right) \times(1+a)=\frac{1+a}{1-e^{-\left(a p_{3}+p_{2}\right) G}}
$$

Where $(1+a)$ represents the length of information packet whether it transmitted successfully or not in the TP cycle ${ }^{[8]}$.

The average length of I event is:

$$
E(I)=E\left(N_{I}\right) \times a=\frac{a}{1-e^{-a p_{1} G}}
$$

The throughput of the new protocol in channel $i$ is:

$$
\begin{aligned}
& S= \frac{E(U)}{E(B)+E(I)} \\
&= \frac{\frac{a p_{1} G e^{-a p_{1} G}}{1-e^{-a p_{1} G}}+\left(a p_{3}+p_{2}\right) G}{1+a} \\
& 1-e^{-\left(a p_{3}+p_{2}\right) G}+\frac{a}{1-e^{-a p_{1} G}}
\end{aligned}
$$

\subsection{The Analysis of System Delay}

First do the following assumptions before analyze the system delay: the monitoring signal can always being transmitted correctly; the time generating monitoring signal can be ignored; $\mathrm{R}$ is the average delay of a packet to be transmitted twice, then $\mathrm{R}$ is formed by four parts: the time that information packet transmitted 1, round-trip propagation delay $a$, and the average retransmission delay $\delta$ [9].

The average delay of information packets for the three-dimensional probability CSMA protocol is:

$$
D=\left[\frac{\frac{(1+a) G}{1-e^{-\left(3 a p_{3}+p_{2}\right) G_{i}}}+\frac{a G}{1-e^{-a p_{1} G_{i}}}}{\frac{a p_{1} G_{i} e^{-a p_{1} G_{i}}}{1-e^{-a p_{1} G_{i}}}+\left(3 a p_{3}+p_{2}\right) G_{i}}-1\right] R+1+\tau_{A}
$$

\subsection{The Analysis of Energy Efficiency}

Assuming the transmitting power of sensor node is $P_{t x}$, receiving power is $P_{r x}$, the power of detection channel is $P_{d d}$.

1) Energy consumption analysis

The detecting duration of all service nodes in the idle time is:

$$
t_{d}(I)=\frac{a^{2}}{2} G E(I) \frac{a}{1-e^{-G P_{1} a}}
$$

Then the sensing consumption in the next $T_{i}$ is:

$$
W_{d}=\left(t_{d}(B)+t_{d}(I)\right) P_{l s m}
$$

Therefore, the average operating power of channel $i$ is:

$$
P(S)=\frac{W_{\mathrm{t}}+W_{\mathrm{rec}}+W_{\mathrm{d}}}{E(B)+E(I)}
$$

2) The life cycle of node

For the terminal nodes, assuming it sleeps 1 at a time, then it sleeps $a$ within time $a$, the average 
power of the node which priority is $l$ in the $\mathrm{N}$ nodes is:

$$
P_{d}=\frac{W_{\mathrm{t}}+W_{\mathrm{d}}}{E(B)+E(I)+\left[q_{2}^{0}+q_{\tau_{A}}^{0} \tau_{A}+a q_{1}^{0}\right] \frac{E(B)}{1+a+\tau_{A}}}
$$

Where, $q_{\tau_{A}}^{0}$ is the definition of the probability that there is no packet to be sent within time $\tau_{A}$ in a transmission period, $q_{\tau_{A}}^{0}=e^{-G P_{3} \tau_{A}}$.

For the aggregation nodes, the average power is:

$$
P_{s}=\frac{W_{r e c}+P_{l s m} \frac{E(I)+E(B)}{T_{s}} a}{2 E(B)+E(I)+\frac{E(I)}{a}}
$$

Since the energy consumption is constant in the transmission process, therefore, for the terminal nodes under the control of MCPDCSMA/ACK protocol, the lifecycle $T_{d}$ is:

$$
T_{d}=\frac{E}{24 \times 365 \times P_{d}+0.1 E}
$$

For the aggregation node, the lifecycle $T_{s}$ is:

$$
T_{s}=\frac{E}{24 \times 365 \times P_{s}+0.1 E}
$$

\section{Simulation}

From the above analysis, the expression of the system throughput under the discrete time threedimensional probability CSMA protocol is got. With the simulation tool-MATLAB R2010a, the simulation results are shown in Figure 2 to Figure 6.

Before the simulation, assume that:

a) The channel is ideal with no noise and interference;

b) The basic unit of the system control clock is $a$, the information packets arrived at time $a$ will transmit at the starting time of the next slot;

c) The channel propagation delay is $a$, the packet length is unit length and is an integral multiple of $a$;

d) The arrival process of the channel satisfy the Poisson process whose independent parameter is $G$, each arrival process on the channel is independent of each other;

e) The $P$ is the probability of the discrete timeslot P-detection CSMA protocol which is proposed by Dongfeng Zhao professor;

f) The packet length is 1 .

g) $a=0.01$

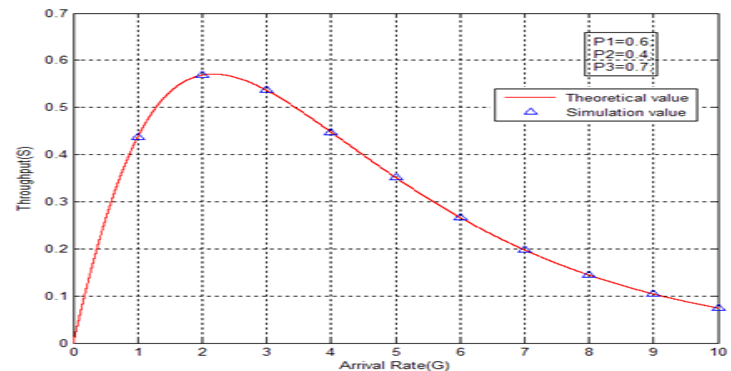

Fig.2. The throughput of the new protocol

In Fig.2, the simulation values of system throughput under the new protocol are consistent with the theoretical ones, verified the correctness of mathematical derivation done before.

When $G$ becomes bigger, the system throughput is increase; especially when $G$ is near 1.5 , the system throughput is very high; next when $G$ is bigger, the system throughput decreases.

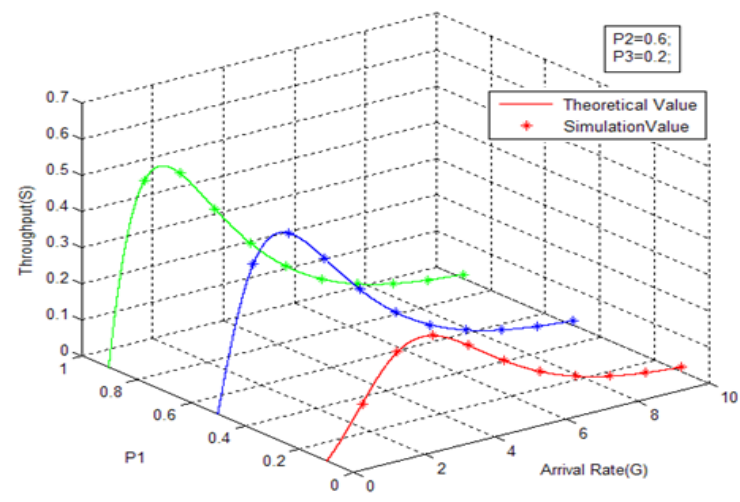

Fig.3. The throughput of new protocol with variable P1

In the Fig.3, the simulation values of system throughput under the new protocol are consistent with the theoretical ones, verified the correctness of mathematical derivation done before.

When $P 1$ becoming bigger, the throughput will increase, especially with small value of $G$; because when the channel is idle, the probability of an information packet sent successfully will increases; the utilization of channel resources is improved.

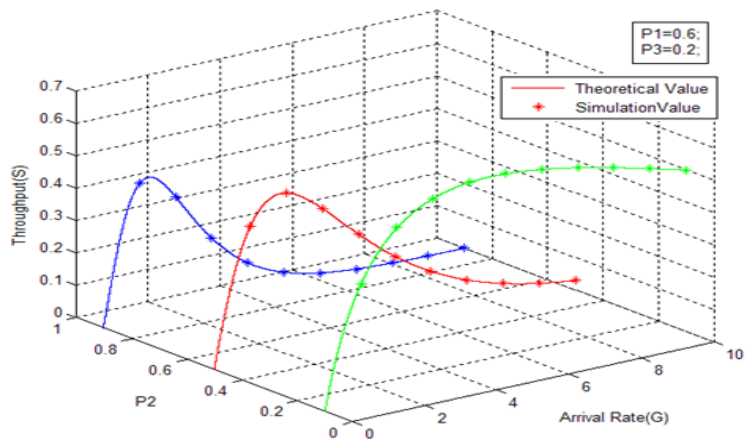

Fig.4. The throughput of new protocol with variable P2

In the Fig.4, the simulation values of system throughput under the new protocol are consistent with the theoretical ones, verified the correctness of mathematical derivation done before.

When $P 2$ becoming bigger, the throughput will decrease; because when the channel is busy sending the packet, if the more new arrival information 
packets the more collisions will be; the utilization of channel resources decreases.

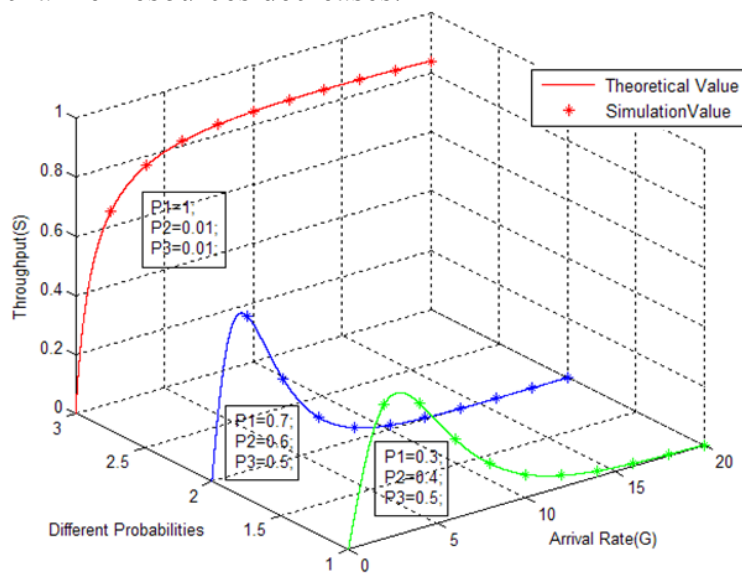

Fig.5. The comparison of system throughput under the new protocol with different probabilities

In the Fig.5, the simulation values of system throughput under the new protocol are consistent with the theoretical ones, verified the correctness of mathematical derivation done before.

From the figure, we can find that changing the variable $P 1, P 2$ and $P 3$ at the same time to get value of the system throughput needed.

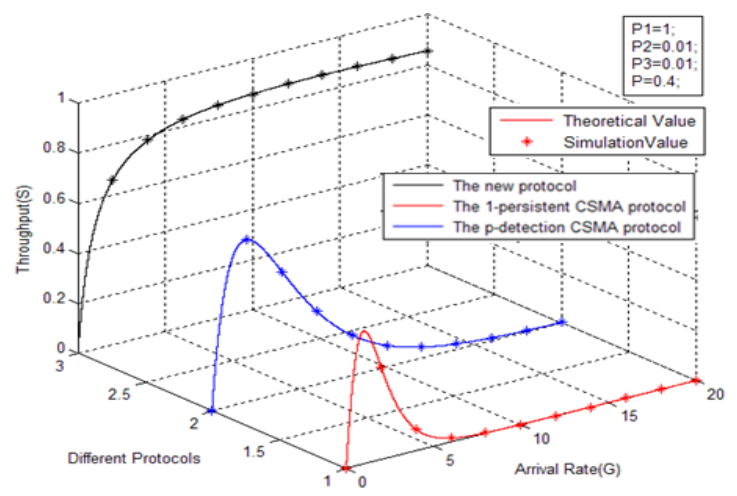

Fig.6. The comparison of system throughput under the different protocols

In the Fig.6, the simulation values of system throughput under the new protocol are consistent with the theoretical ones. When $a=0.01 \quad P=0.4$ $P 1=1 \quad P 2=P 3=0.01$, the throughput of the system under new protocol is bigger than the ones using the non-persistent CSMA protocol or the P-detection CSMA protocol, showing the priority than other protocols. On the whole, throughput of system using three-dimensional probability CSMA protocol can increases to a certain extent.

Through the above figures, we can find that when $a=0.01 P 1=1 P 2=P 3=0.01$, the throughput of the system under new protocol is approaching 1 . If we need the system throughput to get the value, we can set the parameters just as above.

\section{Conclusions}

At present, wireless sensor mainly uses the battery without uncharged ability as the power source. In the present technical conditions, there are still certain difficulties to improve the battery capacity per unit volume. So whether we can make reasonable use of the existing energy resources is the main factors of the network lifetime. The discrete time three-dimensional probability CSMA protocol, using the average cycle method, gets the precise mathematical expressions of system throughput, system delay and energy efficiency by rigorous mathematical derivation. The correctness of the theory is verified through the simulation.

With the continuous development of technical conditions, the environmental protection of the power supply technology such as solar power, wind power generation has gradually mature, use of these skills will also gradually mature. There is reason to believe that in the near future, energy supply problems will no longer become a bottleneck restricting the development of wireless sensor network, wireless sensor network will feed into every aspect of our lives. To the progress of the society as a whole development, it will play a more and more important role.

\section{References}

[1].Jinyang Li, Charles Blake, Douglas S. J. De Couto, Hu Imm Lee, and Robert Morris, Capacity of Ad Hoc Wireless Networks, in the proceedings of the 7th ACM International Conference on Mobile Computing and Networking, Rome, Italy, July 2001.

[2].Yuhong Zhang, Wei Li. Modeling and energy consumption evaluation of a stochastic wireless sensor network[J]. EURASIP Journal on Wireless Communications and Networking, 2012(1): 79-82.

[3].Hongwei Ding, Yingying Guo, Qianlin Liu and Shengjie Zhou. The Multichannel Pd-Csma With 3-Way Handshake Based On Conflict Resolution Algorithm In Wsn. International Journal of Recent Scientific Research Vol. 6, Issue, 4, pp.3714-3718, April, 2015.

[4].Zhao Dongfeng. Study on A New Method for Continuous-time Systems of Random Access Channel [J]. Journal of Electronics, 1999, 21(1): 37-41.

[5].Hung LeHong and J. Fenn. Garnter Report. "Hype Cycle for Emerging Technolo-gies", July 2012. [Online]. Available: http://www.gartner.com/newsroom/id/2124315. [6].Hongwei Ding, Yingying Guo, Yifan Zhao, Shengjie Zhou, and Qianlin Liu. Sensor Lett. 13, 143-146 (2015).

[7].D. Vesset and B. Woo et al. "Worldwide Big Data Technology and Services2012-2015 Forecast," IDC Report 233485, March 2012. [Online]. Available: http://www.idc.com/getdoc.jsp? containerId=233485.

[8].E. A. Brewer."Towards robust distributed systems,"inProc. 19th Annual ACM New Method for the Slotted Access Channel [J]. Journal of Electronics, 1997, 19(6):814-819.

[9].Zhao Dongfeng, Li Bihai, Zheng Sumin. Study on a New Method for the Slotted Access Channel [J]. Journal of Electronics, 1997, 19(6):814-819.

[10].Yi Shang, Hongchi Shi. Flexible Energy Efficient Density Control on Wireless Sensor Networks[J]. International Journal of Distributed Sensor Networks, 2007, 3(1): 101-120.

[11].Muaz Niazi, Amir Hussain (March 2009). "Agent based Tools for Modeling and Simulation of SelfOrganization in Peer-to-Peer, Ad Hoc and other Complex Networks, Feature Issue" (PDF). IEEE Communications Magazine. 47 No.3. Cs.stir.ac.uk. pp. 163-173. 